\author{
MILITARY TECHNICAL COLLEGE \\ CAIRO - EGYPT
}

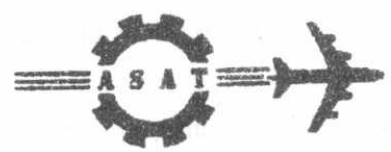

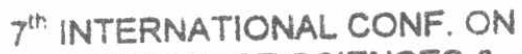
AEROSPACE SCIENCES \& AVIATION TECHNOL.OGY

\title{
Numerical Simulation and Experimental Study on Forced Deflection Nozzles
}

\author{
Cai Timin Yu Shengchun He Hongqing \\ College of Astronautics \\ Northwestern Polytechnical University \\ Xi'an Shaanxi, China, 710072
}

\begin{abstract}
A Compressible thin-layer Navier-stokes solver is developed based on the flux vector splitting method to investigate 3-D flow field of forced deflection nozzle(FDN). 3-D grid generation is accomplished by solving Possion Equation. The Block-Line-Gauss-Siedel relaxation approach is employed to solve the discretization equations with upwind scheme. Numerical results show that FDN is of the ability to compensate the variation of environmental pressure automatically. Firing test experimental study is also conducted for FDN which proves that FDN is feasible and effective. Numerical and experimental results about FDN pressure distribution are in quite good agreement.
\end{abstract}

\section{Introduction}

The conventional propulsive nozzle is an axisymmetric De Laval nozzle, such nozzles have been used successfully for many years in rocket engines, turbojet engiens and ramjet engines. Their advantages mclude efficient design-point performance, high reliability, reasonable cost and a rich background of experience. Its disadvantages include excessive length and weight, inefficient offdesign performance ${ }^{[1]}$. So Forced Deflection Nozzle(FDN) has been considered to overcome these disadvantages ${ }^{[2]}$. A scheme of the FDN is shown in figure 1. Combustion gas is directed radially into the exit cone contour. This concentrates the gas flow along the exit cone wall which facilitates turning the flow back to a direction parallel to the nozzie axis, and resuits in lower divergence loss and a more efficient nozzle. In order to obtain an overall understanding to the FDN, we carried out 3-D numerical simulation for its flow field, and also conducted firing test experimental study.

An efficient relaxation algorithm for the three dimensional thin-layer Navier-Stokes equations is obtained by using upwind differencing for the convective and pressure terms and central differencing tor the viscous shear and heat flux terms. The upwind differencing in the present work is implemented using the flux vector splitting method developed by Van Leer. 3-D grids generation is accomplished by solving Possion Equations according to the Thomas method. The threedimensional flowfields of FDN under different outlet pressures are calculated, the computational results show the fact that the FDN has the ability to compensate automatically the variation of environmental pressure. Firing tests for the FDN and conventional nozzle with same throat and exit areas also show approximately the equal total impulses, which confirms that the FDN is feasible and effective provided the errosion and ablation problem of throat material is well treated. Numerical and experimental results in FDN pressure distribution are in quite good agreement. 


\section{Numerical Simulation}

It is assumed that the flow of FDN is turbulent and is not affected by the body forces. The non-dimensional form of the three-dimensional time-dependent thin-layer Navier-Stokes equations in conservation form and general coordinates is given below ${ }^{|3|}$.

$$
\frac{1}{J} \frac{\partial Q}{\partial t}+\frac{\partial \hat{E}}{\partial \xi}+\frac{\partial \hat{F}}{\partial \eta}+\frac{\partial \hat{G}}{\partial \zeta}-\frac{1}{R \mathrm{e}} \frac{\partial \hat{F}}{\partial \eta}=0
$$

with the equation of state given by $p=(\gamma-1)\left[e-0.5 p\left(u^{2}+v^{2}+w^{2}\right)\right]$

The grids generation method of Thomas ${ }^{(4)}$ is used in present work. Where, $\xi=\xi(x, y, z)$, $\eta=\eta(x, y, z), \zeta=\zeta(x, y, z), J=\partial(\xi, \eta, \zeta) / \partial(x, y, z)$ denotes the Jacobian determinant of coordinates transformation.

The flux splitting method developed by Van Leer ${ }^{t S_{1}}$ is used to discrete the convective and pressure terms. The inviscid fluxes $\hat{E}, \hat{F}$ and $\hat{G}$ are split into $\hat{E}=\hat{F}=$ and $\hat{G}=$ according to the Jacobian matrices as below

$$
\begin{aligned}
& \frac{\partial \hat{E}}{\partial \xi}+\frac{\partial \hat{F}}{\partial \eta}+\frac{\partial \hat{G}}{\partial \zeta}=\delta ; \hat{E}+\delta_{\xi} \hat{E}^{-}+ \\
& \delta_{\eta} \hat{F}^{*}+\delta_{\eta} \hat{F}^{-}+\delta_{;} \hat{G}^{+}+\delta ; \hat{G}^{-}
\end{aligned}
$$

where, $\Delta \xi=\Delta \eta=\Delta \zeta=1, \delta_{\xi}^{-}, \delta_{\eta}^{-}, \delta_{\xi}^{-}$denote backward difference operators and $\delta_{\xi}, \delta_{\eta}, \delta_{\zeta}$ denote forward difference operators. When the local Mach number in the $\xi$ direction $\left.\right|_{M}:<1$, the splittung fluxes can be expressed as

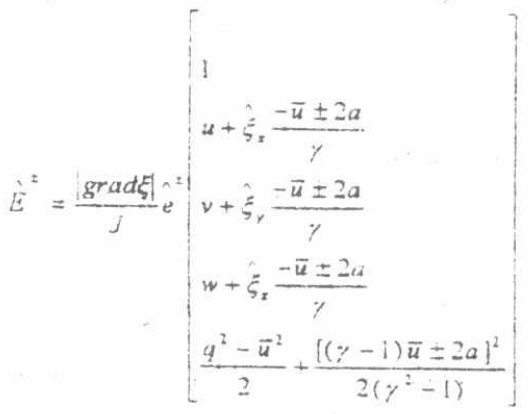

$$
\begin{aligned}
& \ddot{e}_{i}= \pm \frac{p a}{4}\left(M_{i} \pm 1\right)^{2}= \pm \frac{\rho}{4 a}(\bar{u} \pm a)^{2} \\
& \bar{u}=\xi, u+\dot{\xi}_{v} v+\xi, w \\
& \left(\xi_{x}, \xi_{y}, \xi_{z}\right)=\frac{\left(\xi_{x}, \xi_{y}, \xi_{z}\right)}{\text { grad } \xi} \\
& q^{2}=u^{2}+v^{2}+w^{2}
\end{aligned}
$$

when

$$
\begin{aligned}
& u_{;} \geq 1, \hat{E}=\hat{E}, \hat{E}=0 \\
& w_{;} \leq-1, \hat{E}=0 \cdot \hat{E}=\hat{E}
\end{aligned}
$$

The splitting fluxes expressions of $F$ and $\hat{G}$ are similar to the equation (3). The split differences are represented as a flux balance across a cell, for example 


$$
\begin{aligned}
& \delta_{\xi} \hat{E}^{+}+\delta_{\xi}^{+} \hat{E}^{-}=\left[\hat{E}^{-}\left(Q^{-}\right)+\hat{E}^{-}\left(Q^{+}\right)\right]_{i+1 / 2} \\
& -\left[\hat{E}^{+}\left(Q^{-}\right)+\hat{E}^{-}\left(Q^{+}\right)\right]_{i-1 / 2}
\end{aligned}
$$

The notation $\hat{E}^{+}\left(Q^{-}\right)$denotes $\hat{E}^{+}$evaluated at $Q^{-}$and $Q^{ \pm}$represent upwind interpolations to cell interfaces

$$
\begin{aligned}
& Q_{i+1 / 2}^{-}=Q_{1}+\frac{\varphi}{4}\left[\left(1-\kappa_{x}\right) \nabla_{x}+\left(1+\kappa_{x}\right) \Delta_{x}\right] Q_{i} \\
& Q_{i+1 / 2}^{+}=Q_{i+1}-\frac{\varphi}{4}\left[\left(1-\kappa_{x}\right) \Delta_{x}+\left(1+\kappa_{x}\right) \nabla_{x}\right] Q_{i+1}
\end{aligned}
$$

where, the parameters $\kappa$ and $\varphi$ determine the accuracy of the spatial differencing.

Discretization of Eq.(1) in space and also in time using the Euler implicit method results in:

$$
\frac{\Delta Q}{J \Delta t}=R^{n+1}(Q), \Delta Q=Q^{n+1}-Q^{n}
$$

where the superscript $\mathrm{n}$ refers to the known time level. $\mathrm{R}(\mathrm{Q})$ is the residual

$$
\begin{aligned}
& R(Q)=-\delta_{\xi}^{-} \hat{E}^{+}-\delta_{\xi}^{+} \hat{E}^{-}-\delta_{\eta}^{-} \hat{F}^{+}-\delta_{\eta}^{+} \hat{F}^{-} \\
& -\delta_{\zeta}^{-} \hat{G}^{+}-\delta_{\zeta}^{+} \hat{G}^{-}+\frac{1}{\operatorname{Re}} \delta_{\eta} \hat{F}_{V}
\end{aligned}
$$

Linearizing the residual about the known time level and neglecting the variation of viscosity in the linearization, eq. $(7)$ can be written as

$$
\begin{aligned}
& {\left[\frac{I}{J \Delta t}+\delta_{\xi}^{-\hat{A}^{+}}+\delta_{\xi}^{+} \hat{A}^{-}+\delta_{\eta}^{-} \hat{B}^{+}+\delta_{\eta}^{+} \hat{B}^{-}+\right.} \\
& \left.\delta_{\zeta} \hat{C}^{+}+\delta_{\zeta}^{+} \hat{C}^{-}-\frac{1}{\mathrm{Re}} \delta_{\eta} \hat{B}_{\nu}\right] \Delta Q=R^{n}(Q)
\end{aligned}
$$

where $\hat{A}^{ \pm}, \hat{B}^{ \pm}, \hat{C}^{ \pm}$and $\hat{B}_{v}$ are Jacobian matrices of $\hat{E}^{ \pm}, \hat{F}^{ \pm}, \hat{G}^{ \pm}$and $\hat{F}_{\nu} . \mathrm{I}$ is the $5 \times 5$ identity matrix. When a higher order upwind spatial discretization for $R(Q)$ is used, with a consistent spatial discretization of the left-hand of eq.(9), a vector equation may be written for each cell in the domain, given by:

$$
\begin{aligned}
& \bar{A} \Delta Q_{i-2}+\bar{B} \Delta Q_{i-1}+\bar{C} \Delta Q+\bar{D} \Delta Q_{i+1}+\bar{E} \Delta Q_{i+2}+\bar{F} \Delta Q_{j-2}+\bar{G} \Delta Q_{j-1}+ \\
& \bar{H} \Delta Q_{j+1} \bar{I} \Delta Q_{j+2}+\bar{J} \Delta Q_{k-2}+\bar{K} \Delta Q_{k-1}+\bar{L} \Delta Q_{k+1}+\bar{M} \Delta Q_{k+2}=R(Q)
\end{aligned}
$$

where $\bar{A}-\bar{M}$ are linear combinations of the flux Jacobian matrices, and $\bar{C}$ contains the time term. In the above, and throughout the remainder of this paper, a superscript ' $n$ ' and subscript ' $i$ ', 'j' or ' $k$ ', are dropped for notational convenience.

Based on the diagonally dominant property of coefficient matrices arising from such discretizations, the relaxation algorithm is implemented by sweeping back and forth across the mesh, as dictated by stability considerations. The down left to upper right sweep can be written as

$$
\begin{aligned}
& (\bar{F}, \bar{G}, \bar{C}, \bar{H}, \bar{I}) \Delta Q=R(Q)-\bar{A} \Delta Q_{i-2} \\
& -\bar{B} \Delta Q_{i-1}-\bar{J} \Delta Q_{k-2}-\bar{K} \Delta Q_{k-1}
\end{aligned}
$$

and the upper right to down left sweep is written as

$$
\begin{aligned}
& (\bar{F}, \bar{G}, \bar{C}, \bar{H}, \bar{I}) \Delta Q=R(Q)-\bar{D} \Delta Q_{i+1} \\
& -\bar{E} \Delta Q_{i+2}-\bar{L} \Delta Q_{k+1}-\bar{M} \Delta Q_{k+2}
\end{aligned}
$$

For increasing the overall convergence rate, the boundary conditions are treated implicitly and combined into the differenced equations of the interior grid points. No-slip and isothermal conditions are given for the wall boundary. The supersonic inflow boundary conditions are given, for the discrete throats are well distributed in the circumference. The symmetric conditions are used on the two sides of the computational region. The outflow boundary which is different from the 
conventional nozzle is supersonic near the wall and is subsonic near the axis, so the supersonic and subsonic flow exist commonly, each point is need to distinguish in each time step. For the supersonic points, all the variables are extrapolated from the interior flow field. For the subsonic points, pressure condition is treated by the formula of $\partial p / \partial x=0$, and the rest variables are extrapolated also.

The generated grids are shown in figure 2, the grid points are well-distributed in the direction of axis and circumference. In order to assure the accuracy of numerical calçulation in the boundary layer, the grid points are concentrated near the wall in the radial direction.

The numerical simulation is made for the flowfield in divergent section of FDN under different outside pressures using the above algorithm.

The pressure distribution is shown in figure 3. The non-dimensional pressure(outflow pressure/critical pressure) is 0.012 . The gas flowed out from the discrete throats expands rapidly. In the base of the throat section, an excessive expansion region is generated. The pressure along the axis is nearly equal to the outside pressure.

Figure 4 . is the velocity vector plots at different outflow pressures. When the outside pressure is greater, the gas is concentrated to the wall and the velocity in axis is very small, the expansion region becomes great with the decreasing of the outside pressure. The fact proves that the FDN has the ability to compensate the variation of the outside pressure.

The variation of the wall pressure along the axis is shown in figure 5. The pressure decreases rapidly at the begin and is almost unvaried near the exit, this is in good agreement with the test results.

\section{Experimental study}

The experiments for FDN include the firing tests compared with the conventional cone nozzle under the condition of same throat area and same exit area, two kinds of nozzle are shown in Fig.6. Firing test is conducted on a small test stand with calibration in situ, thrust and pressure are measured by sensors and data acquisiton system, the accuracy of measurement is about $0.5 \%$. The measured thrusts are shown in Fig.7. The result that the total impulses of two kinds of nozzle are equal approximately can confirm that the FDN is feasible provided the errosion and ablation problem of throat material is well treated, molybdenum is used to make the throat part so that errosion and ablation of material can be prevented during the firing test of FDN. Pressure at points A, B, C, D (see Fig.6) are also measured during FDN firing tests, $P_{A}, P_{B}, P_{C}$ are shown in Fig. 5 with " $O$ ". $P_{D}$ which is measured at the base of throat section is always a little bit lower than outside environmental pressure(see Fig. 8), this may be resulted by the suddenly excessive expansion at the base of throat section near exit of throat hole and the extraction of high speed gas flow in the divergent section of nozzle creating some vacuum. This result is also coincide with the numerical simulation.

To further study the FDN performance with different deflection angles of throat hole, a case of $30^{\circ}$ deflection angle is experimented with other conditions unchanged. Result shows that the thrust with $45^{\circ}$ deflection angle is a little bit higher than that of $30^{\circ}$, corresponding specific impulse increased by $0.5 \%$. 


\section{Summary}

Numerical simulation and experimental study prove that FDN is feasible and effective, provide the errosion and ablation problem of throat material is well treated. FDN has the ability to compensate the variation of outside environmental pressure which can keep high off-design performance. FDN can be made in shorter length and then reduce its weight. We believe that FDN is of good prospects in application.

\section{REFERENCES}

[1] Marcum, D.L. and Hoffman, J.D., "Cal-culation of Three-Dimensional Inviscid Flowfields in Propulsive Nozzles with Centerbodies", ALAA paper 86-0449, Jan. 1986.

[2] Mcparland, G.G., Bennett, D.R., Coon, J.W., Mittermaier,N.P., "Integrated Stage Concept System Study Result", AIAA paper 86-1581, June, 1986.

[3] Schroder, W. and Hartman, G., "Implicit Solutions of Three-Dimensional Viscous Hypersonic Flows", Computers Fluids, Vol.21, No.1, 1992. pp.109-132.

[4] Thomas, P.D., "Composite Three-Dimensional Grids Generated by Elliptic Systems", ALAA Journal, Vol.20, No.9, Sep, 1982. pp.1195-1202.

[5] Thomas, J.L., Van Leer , B. , Walters, R.W., "Implicit Flux-Split Schemes for the Euler Equations", ALAA paper 85-1680.

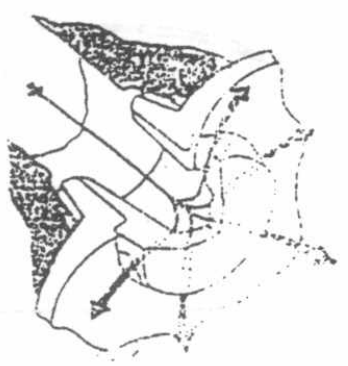

Figure 1. Forced Deflection Nozzle

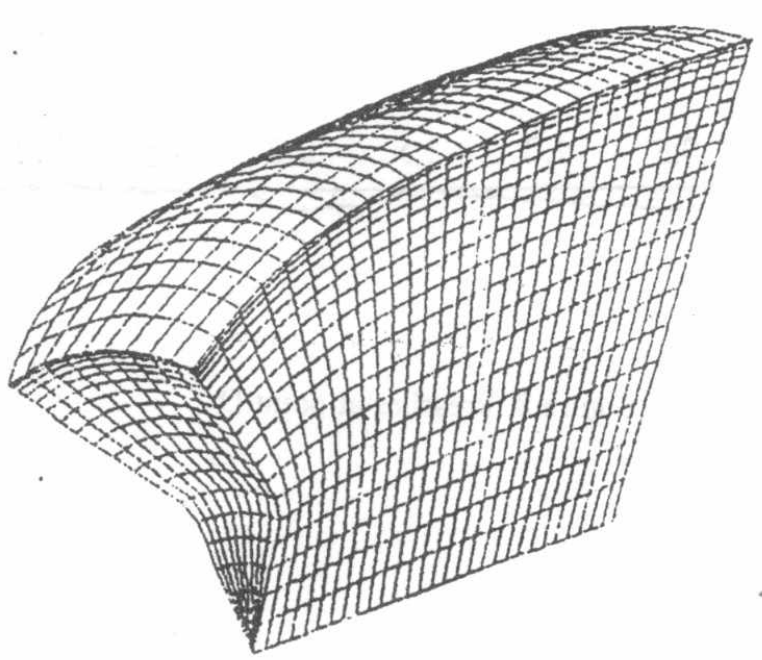

(b) $\mathrm{p}=0.01$

Figure 4. Velocity field in central plane

Figure 2. Three-dimensional grid for FDN 


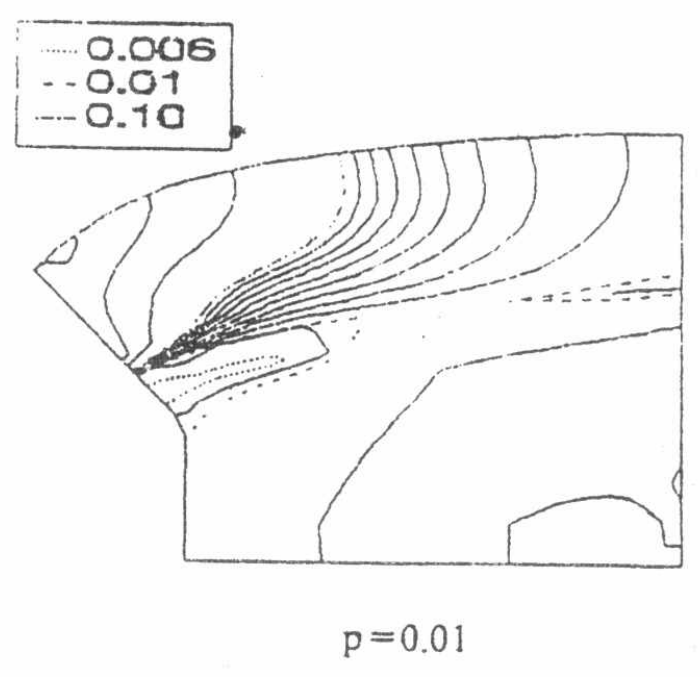

Figure 3. Pressure contour in central plane

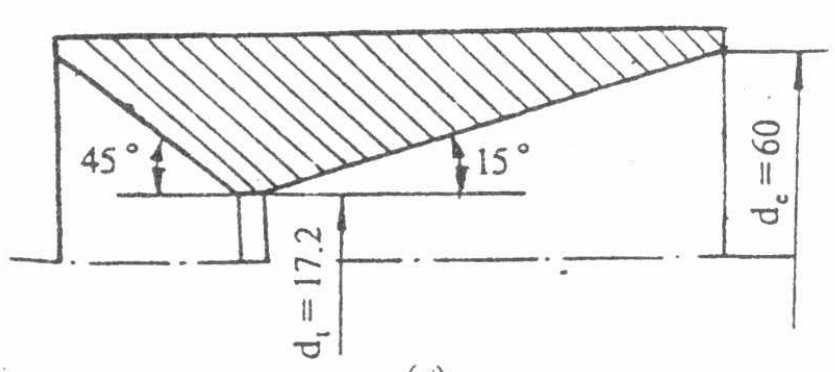

(a)

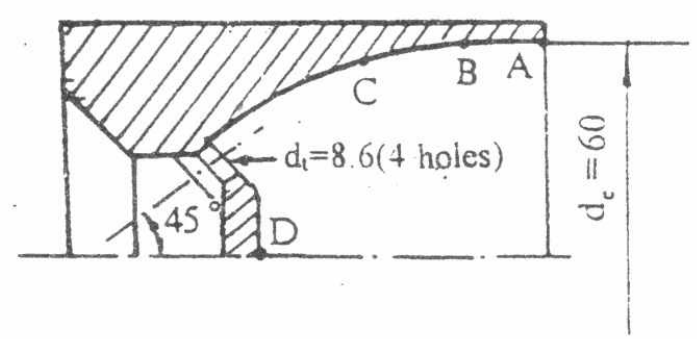

(b)

Figure 6. Cone nozzlc (a) and FDN(b)

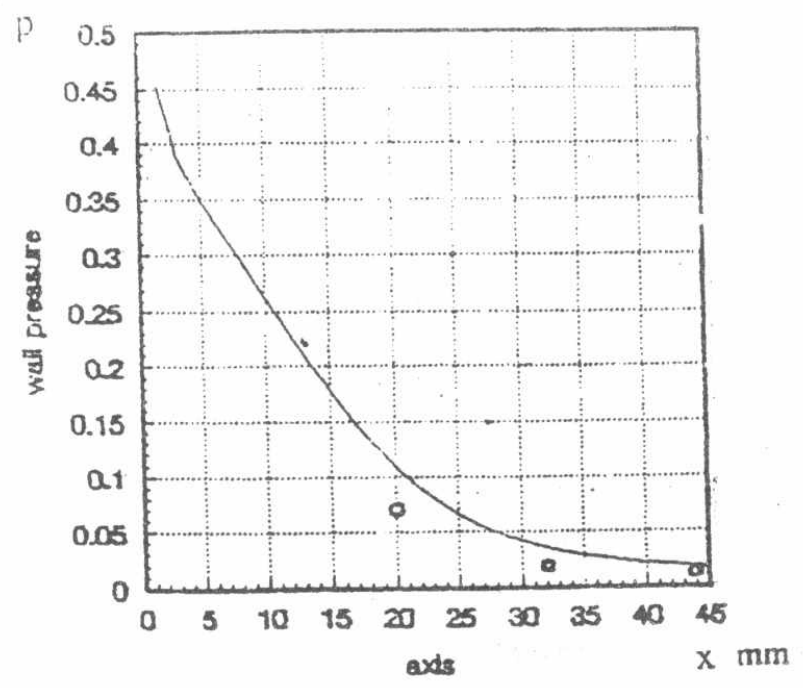

$$
F=1 / 83.5
$$

Figure 5. Wall pressure along axis

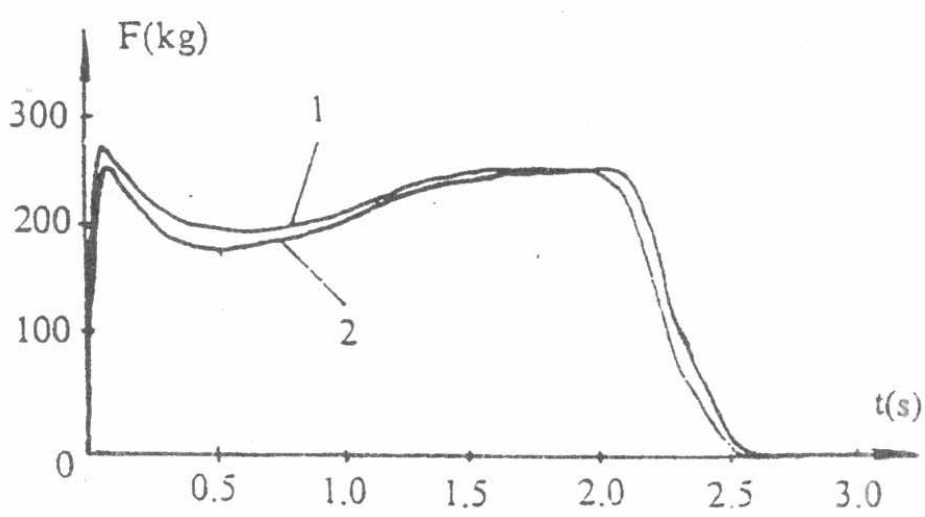

Figure 7. Thrust curves of cone nozzle(1) and FDN(2)

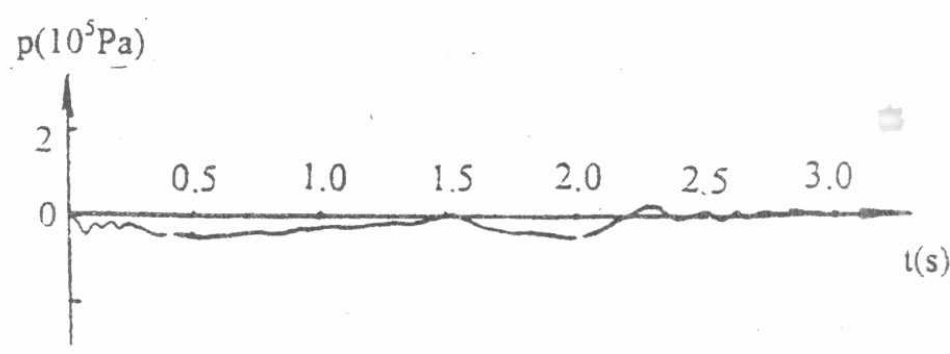

Figure 8. Pressure at point $D$ 